\title{
New generation of measurements and model developments on nuclide production in spallation reactions
}

\author{
K.H. Schmidt ${ }^{\mathrm{a}}$ for the CHARMS collaboration \\ Gesellschaft für Schwerionenforschung mbH, 64291 Darmstadt, Germany
}

\begin{abstract}
With its powerful installations, GSI, Darmstadt, provides unique conditions for fundamental and applied research on a variety of subjects in nuclear physics and other fields with heavy-ion beams of stable and radioactive species. Intensive work on nuclear data is being made, e.g., on nuclear masses, half lives, nuclide yields from lowenergy fission, and nuclide production in spallation reactions. The interest in spallation reactions with primary proton energies around $1 \mathrm{GeV}$ is motivated by the accelerator-driven system, dedicated to the incineration of nuclear waste. An innovative experimental method has been developed, based on inverse kinematics, which allowed for the first time to identify all reaction residues in-flight, using the high-resolution magnetic spectrometer FRS, and thus to determine full nuclide distributions. It also gives direct access to the reaction kinematics. During the last years, an experimental campaign has been carried out in a Europe-wide collaboration, investigating the spallation of several nuclei ranging from ${ }^{56} \mathrm{Fe}$ to ${ }^{238} \mathrm{U}$. Complementary experiments were performed with a full-acceptance detection system, yielding total fission cross sections with high precision. Recently, another detection system using the largeacceptance ALADIN dipole and the LAND neutron detector was introduced to measure light particles in coincidence with the heavy residues. Another intense activity was dedicated to develop codes which cover the nuclear reactions occurring in an ADS in their full energy range and in all target materials involved. Most effort was invested in modelling the later de-excitation stage of the reaction, which is responsible for the salient features observed in the residual nuclide distributions due to the different possible de-excitation paths like evaporation of nucleons, light charged particles and intermediate-mass fragments, fission and multi-fragmentation. The future FAIR facility will provide primary beams with higher intensities and higher energies, a considerably extended variety of secondary beams, and a number of new installations, e.g., powerful magnetic spectrometers, storage rings and colliders. A new generation of experiments on fission-fragment yields with tagged photons using secondary beams is described as an example for future developments.
\end{abstract}

\section{Introduction}

GSI, Darmstadt, the major European research centre for experiments with heavy-ion beams of stable and radioactive nuclides, provides unique conditions for fundamental and applied research on a variety of subjects in nuclear physics and other fields. Even more powerful installations will be available when the FAIR facility will be in operation. Optimum conditions can also be found for research on several types of nuclear data for nuclear technology, radioprotection, astrophysics and other applications. Intensive work on nuclear data is presently being made, e.g., on nuclear masses [1], half lives [2], nuclide yields from low-energy fission [3,4], and nuclide production in spallation reactions. This latter subject that is motivated by the plans for the construction of an accelerator-driven system (ADS) and for the design of the EURISOL facility [5] forms the major part of this contribution.

Often, systematic collections of experimental data cannot cover the full variety of data requested, and nuclear-model calculations are needed to complement the experimental data bases. This is particularly true for the nuclide production in spallation reactions with all relevant target materials and projectile energies. Therefore, also in the field of model developments for the description of spallation reactions, intensive work [6] has been performed at the GSI laboratory.

${ }^{a}$ Presenting author, e-mail: k.h.schmidt@gsi.de

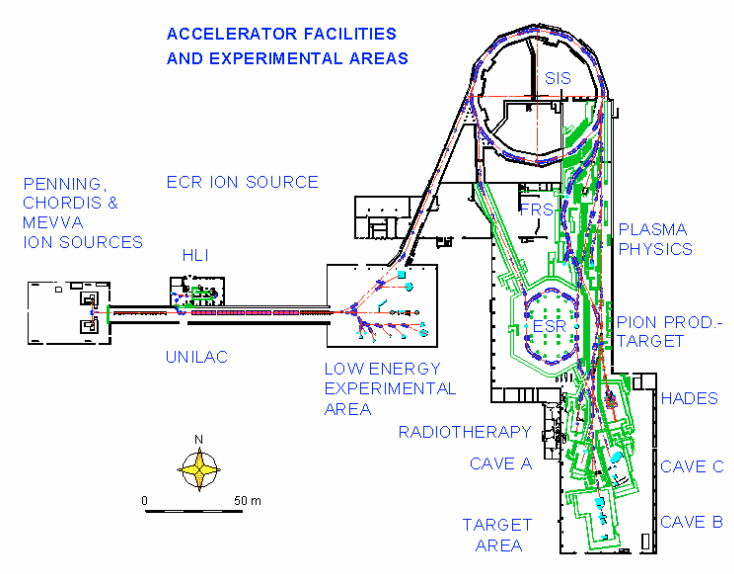

Fig. 1. Installations of the GSI facility.

\section{Installations of GSI}

The basic installation of GSI is the accelerator complex with the UNILAC linear accelerator and the SIS-18 synchrotron, which is capable to provide beams of any stable nuclide up to $18 \mathrm{Tm}$, corresponding to energies of $1 A \mathrm{GeV}$ or higher. The accelerators are complemented by the fragment separator FRS [7], which separates the residues produced in projectilefragmentation reactions, and thus provides a large variety of 
Table 1. List of key reactions studied at the fragment separator.

\begin{tabular}{lll}
\hline projectile & target & $\begin{array}{l}\text { energy in } \\
\text { projectile frame }\end{array}$ \\
\hline${ }^{238} \mathrm{U}$ & $\mathrm{p}, \mathrm{d}$ & $1 \mathrm{GeV}, 2 \mathrm{GeV}$ \\
${ }^{208} \mathrm{~Pb}$ & $\mathrm{p}, \mathrm{d}$ & 0.5 to $2 \mathrm{GeV}$ \\
${ }^{197} \mathrm{Au}$ & $\mathrm{p}$ & $0.8 \mathrm{GeV}$ \\
${ }^{136} \mathrm{Xe}$ & $\mathrm{p}$ & 0.2 to $1 \mathrm{GeV}$ \\
${ }^{56} \mathrm{Fe}$ & $\mathrm{p}, \mathrm{d}$ & 0.3 to $1.5 \mathrm{GeV}$ \\
\hline
\end{tabular}

secondary beams of radioactive species in the same energy range as the primary beams. Dedicated detectors and the largeacceptance dipole magnet ALADIN, mounted in Cave C, as well as the storage ring ESR are important experimental installations for the research on nuclear data.

\section{Experiments on spallation reactions}

Traditionally, experiments on spallation reactions have been performed by bombarding the material of interest by the highenergetic light projectiles. However, this approach has a severe drawback: the spallation residues are produced with very low kinetic energies, such that most of them do not escape from the target. Information on the residue production was obtained by recording the gamma activity after irradiation. However, short-lived nuclides decay, before they can be measured. Using the installations of GSI, Darmstadt, the reaction kinematics could be inverted. The nucleus of interest was used as a projectile, impinging on a liquid hydrogen target. This novel experimental approach allowed for the first time to identify all reaction residues in-flight. It also gives direct access to the reaction kinematics.

\subsection{Nuclide production yields and velocity distributions}

Since several years, an experimental campaign is being pursued in a French-Spanish-German collaboration on measuring the individual nuclide yields in spallation reactions for a number of key reactions. For this purpose, the fragment separator is used as a high-resolution magnetic spectrometer, see figure 2 . All spallation residues are identified in nuclear charge $Z$ and mass number $A$. In addition, their velocity distributions are determined, which are sensitive to the production mechanism.

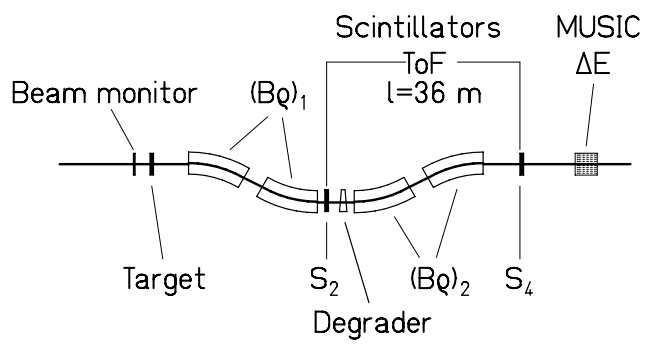

Fig. 2. Schematic view of the fragment separator and the most important detector equipment.

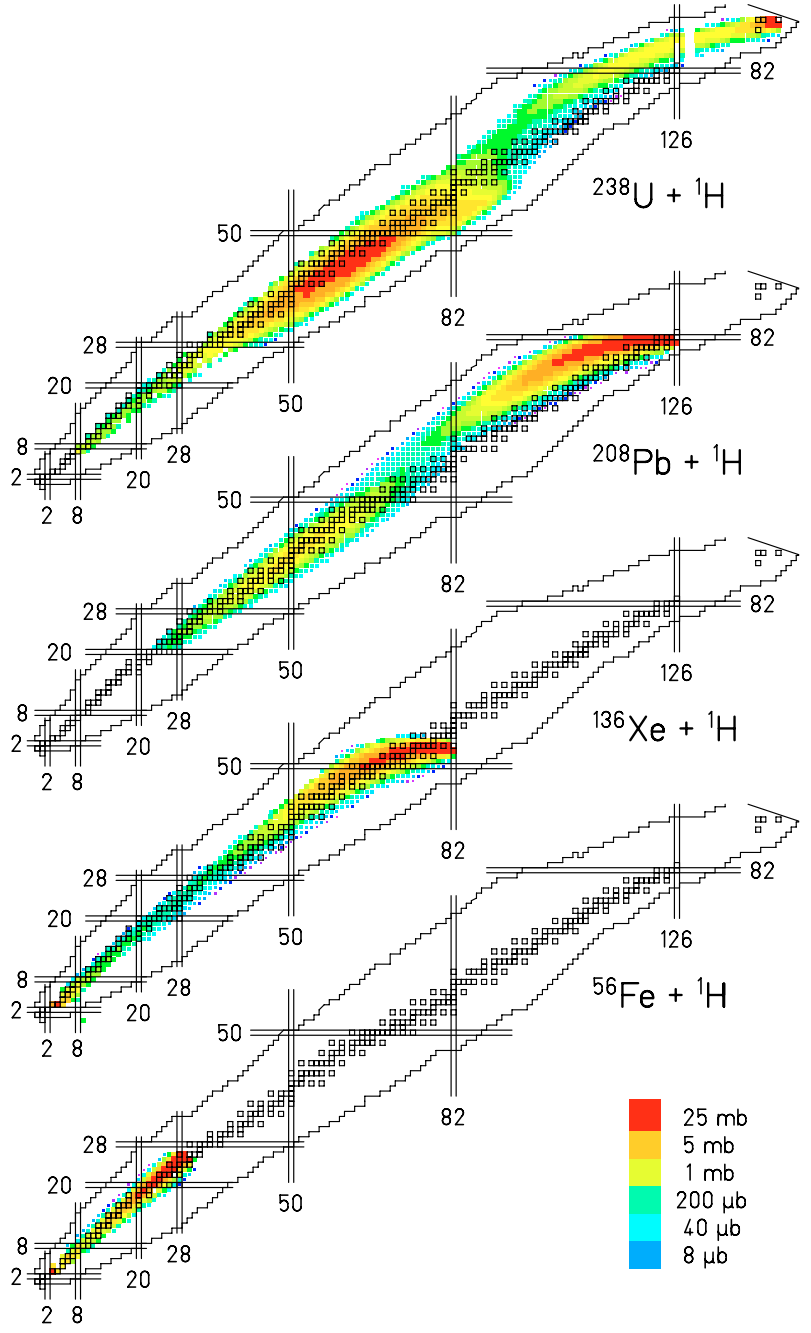

Fig. 3. Overview on the nuclide production cross sections measured at the fragment separator for the four systems indicated at the energy of $1 \mathrm{AGeV}$ on a chart of the nuclides. The colours indicate the production cross sections as defined in the colour scale.

The spallation reactions of a series of projectiles have been studied over an energy range between 0.2 and $2 \mathrm{GeV}$, counted in the frame of the heavy projectile, see table 1 . The measured nuclide cross sections at the energy of $1 \mathrm{GeV}$ are shown in figure 3. Some systematic trends can be observed. While fission is dominant for ${ }^{238} \mathrm{U}$, it contributes only little for ${ }^{208} \mathrm{~Pb}$ and practically disappears for the lighter systems. The spallation-evaporation residues mostly populate the elements close to the projectile and die out around 15 elements below. When covered in the experiment, also sizeable production of light and intermediate-mass fragments is observed, leading to a continuous population of all elements below the projectile. Also some production up to 2 elements above the projectile by charge-pickup reactions is observed.

The longitudinal velocity distributions of these fragments were determined as well with high precision. Figure 4 shows the result for light fragments produced with a ${ }^{136} \mathrm{Xe}$ beam. By including data measured with heavier targets, the bombarding energy in the centre-of-mass systems varies over a large range. 


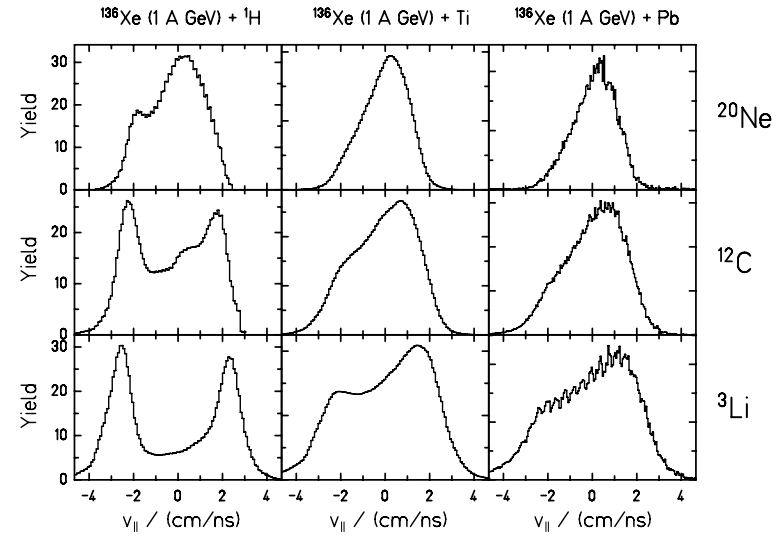

Fig. 4. Longitudinal velocity distributions of light projectile fragments in the projectile frame emitted with transversal angles below $15 \mathrm{mr}[8,9]$. The bombarding energies of the three systems indicated are $1,35.5$ and $82.2 \mathrm{GeV}$, respectively.

The measured longitudinal velocity distributions for these light and intermediate-mass fragments have characteristic shapes, consisting of a double-humped and a single-humped component. The relative weight of the double-humped distribution, which is consistent with a binary decay of a heavy system close to the projectile, decreases with increasing bombarding energy and increasing fragment mass. The singlehumped distribution is consistent with a multifragmentation process. Thus, the longitudinal velocity distributions allow distinguishing these two processes, which both populate the lower part of the U-shaped mass distribution.

\subsection{Total fission cross sections}

The angular acceptance of the fragment separator covers all or at least great part of the spallation-evaporation residues. Spallation-fission products, however, are only transmitted when the fission direction is close to the beam direction, and thus the transmission losses are important. Therefore, a fullacceptance set-up was used to determine the total spallationfission cross sections with higher precision, see figure 5.

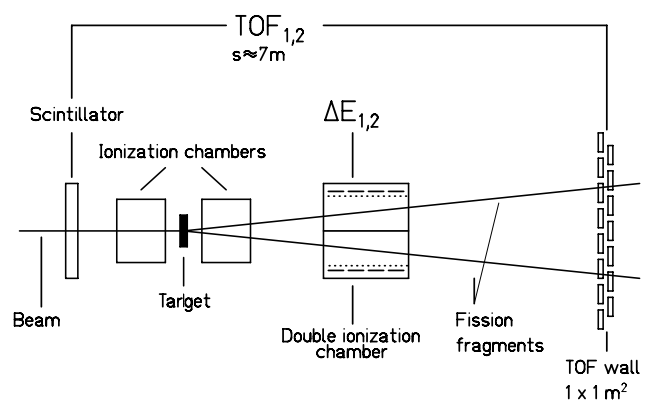

Fig. 5. Set up for full-acceptance measurements of total spallationfission cross sections.

The target is surrounded by two ionization chambers that allow discriminating reactions in other layers. The double ionization chamber and the TOF wall register the two fission fragments in coincidence.
Table 2. Total fission cross sections in mb determined with the fullacceptance set up compared with previous results. The energy is given in the frame of the heavy nucleus. For the values given in green*, the beam energies were slightly different.

\begin{tabular}{|c|c|c|c|c|}
\hline reaction & $\begin{array}{l}\text { full- } \\
\text { acceptance } \\
\text { set up [12] }\end{array}$ & FRS & $\begin{array}{l}\text { Prokofiev } \\
{[10]}\end{array}$ & $\begin{array}{l}\text { Kotov } \\
\text { et al. [11] }\end{array}$ \\
\hline${ }^{208} \mathrm{~Pb}+{ }^{1} \mathrm{H}$ & & $232 \pm 33$ & & \\
\hline $500 \mathrm{MeV}$ & $140 \pm I$ & [13] & 112 & \\
\hline $\begin{array}{l}{ }^{208} \mathrm{~Pb}+{ }^{2} \mathrm{H} \\
1000 \mathrm{MeV}\end{array}$ & $203 \pm 9$ & & & \\
\hline $\begin{array}{l}{ }^{238} \mathrm{U}+{ }^{1} \mathrm{H} \\
545 \mathrm{MeV}\end{array}$ & $1490 \pm 100$ & & 1360 & $1491 \pm 78 *$ \\
\hline $\begin{array}{l}{ }^{238} \mathrm{U}+{ }^{1} \mathrm{H} \\
935 \mathrm{MeV}\end{array}$ & $1550 \pm 100$ & $\begin{array}{l}1530 \pm 200 * \\
{[14]}\end{array}$ & 1270 & $1489 \pm 64 *$ \\
\hline
\end{tabular}

The available results are compared in table 2 with the systematics of Prokofiev [10], and with a recent elaborate experiment [11] performed in normal kinematics. The sum of the individual fission-fragment cross sections measured at the FRS for ${ }^{238} \mathrm{U}+{ }^{1} \mathrm{H}$ are consistent, while there is a discrepancy for ${ }^{208} \mathrm{~Pb}+{ }^{1} \mathrm{H}$, which is not yet understood.

\subsection{Light-charged-particle emission}

Recently, the SPALADIN set up has been developed, allowing for the detection of heavy spallation residues in coincidence with light particles $[15,16]$. The SPALADIN set up (figure 6) is based on the large-acceptance dipole magnet (ALADIN) coupled with a multitrack and multisampling time projection chamber (TPC MUSIC IV).

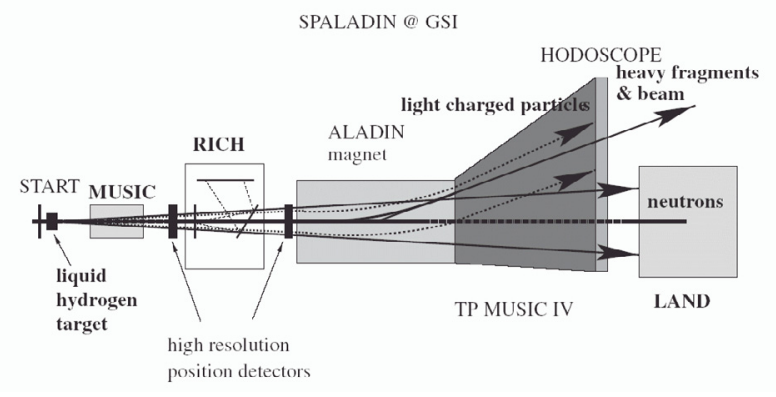

Fig. 6. Schematic view on the SPALDIN experimental set up.

This more exclusive experiment aims to separate the intranuclear cascade phase from the de-excitation phase of the spallation reaction.

\section{Model developments}

The ABLA code, developed at GSI, has proven to be quite successful in modelling the evaporation and the major fission processes in the de-excitation stage of the spallation reaction [17]. However, the production of intermediate-mass 
fragments was severely underestimated [18], because very asymmetric binary emission and simultaneous break-up were not considered. These processes have been included in the new version ABLA07. Also the treatment of the dynamics of the fission process has been improved.

\subsection{Extension of evaporation to heavier masses}

As already discussed by Moretto [19], there is a finite probability for binary mass splits covering the mass range between the major, predominantly symmetric fission, and the evaporation of nucleons and light charged particles. The characteristics of these processes is a continuous transition between evaporation and fission. Previous approaches treat these processes either as very asymmetric fission (e.g., GEMINI [20]) or as evaporation of fragments (e.g., GEM2 [21]). In the new version of the code (ABLA07), an extension of evaporation to higher masses, up to the Businaro-Gallone maximum has been implemented. The emission barriers are based on a realistic nuclear potential [22], leaving no adjustable parameter. In addition, the angular momentum is treated explicitly in the evaporation process. More details can be found in refs. $[6,23]$.

\subsection{Multifragmentation}

If the excitation energy of a system exceeds a certain limit, the yields of intermediate-mass fragments increase, and the emission times are too short to be compatible with the assumption of sequential emission. Also the kinematic properties show clearly specific features (see fig. 4). Different theoretical approaches and the experimental data are consistent with a mass distribution following a power law with an exponent of about 2. Simultaneous break-up has been implemented in ABLA07 for initial excitation energies above $\mathrm{E} / \mathrm{A} \approx 3 \mathrm{MeV}$ following this idea. This value can easily be reached, e.g., in the spallation of light nuclei like iron by $1 \mathrm{GeV}$ protons. The fragments are produced with a freeze-out temperature of 5.5 MeV. More details are again given ref. [6]

\subsection{Fission dynamics}

The treatment of fission dynamics has been implemented in a more consistent way. The initial deformation of the prefragment after the INC phase is considered when calculating the fission transients. This leads to appreciably shorter fission delays in the spallation-fission reactions of nuclei with prolate deformation. Particle emission on the path from saddle to scission and after scission are calculated explicitly. More details are given in ref. [4].

\subsection{Structural effects in fission}

The macroscopic-microscopic approach developed in ABLA, which models the influence of shell effects on the fission process, has been refined. The features of multi-modal fission of all actinides from energies around the fission barrier up to higher energies, at which shell effects disappear, are well

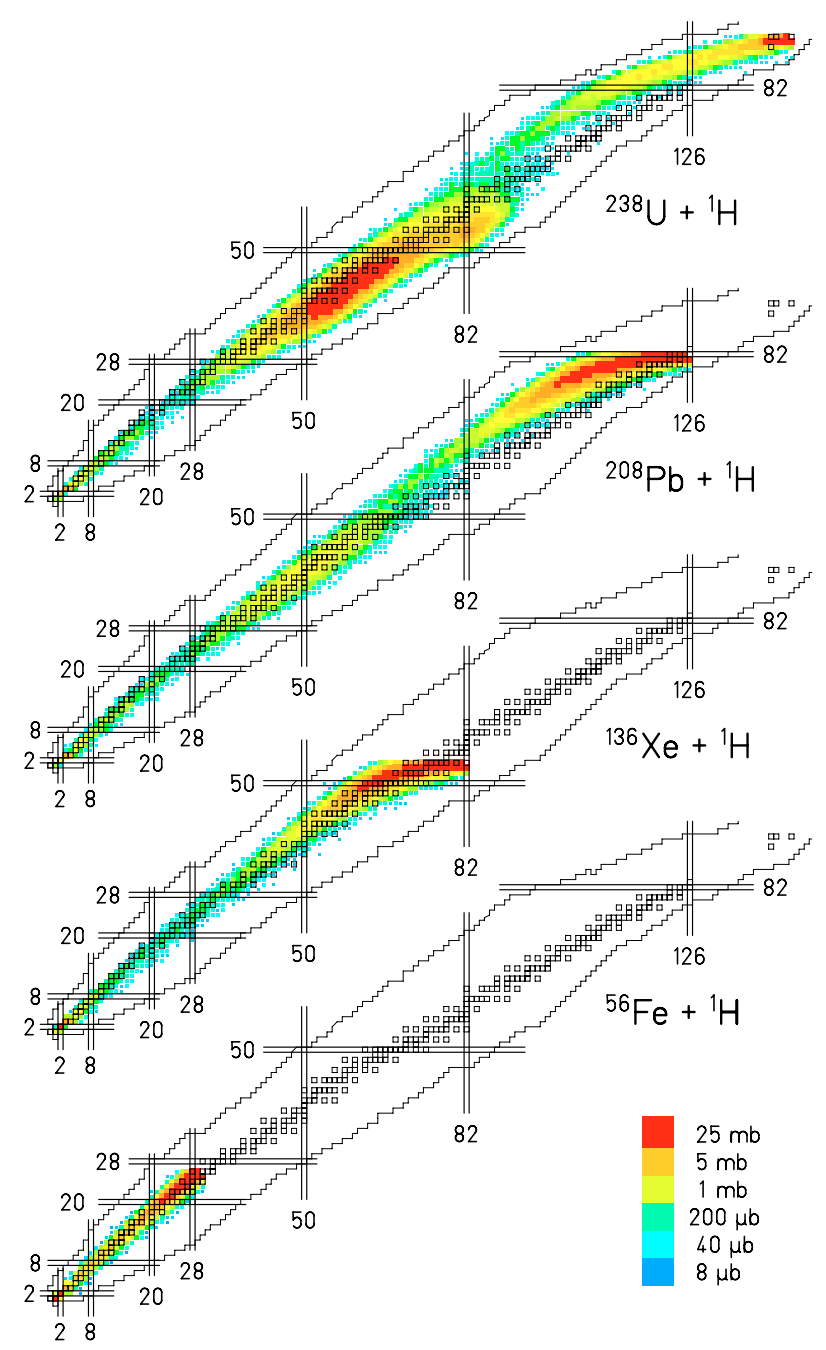

Fig. 7. Overview on the nuclide production cross sections calculated with ABRABLA07 for the four systems indicated at the energy of $1 \mathrm{AGeV}$ on a chart of the nuclides. The colours indicate the production cross sections as defined in the colour scale.

described with a common set of parameters. More details are given in ref. [4].

A good description of the fission process for many systems in an extended region of the chart of the nuclides and over a large excitation-energy range is mandatory for a realistic prediction of spallation-fission residue cross sections, because they result from a superposition of many fissioning systems.

\section{Benchmark}

As a benchmark, the nuclide distributions of the systems shown in figure 3 are compared in figure 7 with ABRABLA07. In this code, the collision phase is described in the abrasion formalism by ABRA07 [24]. The results demonstrate a high degree of similarity with the experimental data. The features of spallation-evaporation residues, spallation-fission products and intermediate-mass fragments, mostly determined by ABLA07, resemble the experimental results. It may be 
concluded that the variations of the nuclide yields with the mass of the system are well reproduced by ABRABLA07.

The calculated variation of the spallation process with bombarding energy can be compared with excitation functions of specific nuclides measured in activation experiments, which cover a large energy range with an important number of steps. Such a comparison has been performed by Titarenko et al. [25], proving that already the previous version of ABLA reproduced the heavy spallation products and the yields in the main fission region with good quality.

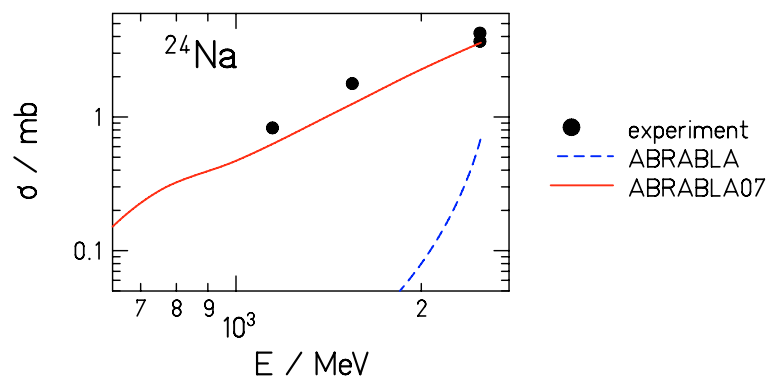

Fig. 8. Measured excitation function of ${ }^{24} \mathrm{Na}$ produced in the reaction ${ }^{209} \mathrm{Bi}+\mathrm{p}[25]$ compared with the previous ABRABLA version and the new code ABRABLA07.

Figure 8 allows a closer view on the threshold behaviour in the production of intermediate-mass fragments by comparing the excitation function of ${ }^{24} \mathrm{Na}$ from the reaction ${ }^{209} \mathrm{Bi}+\mathrm{p}$, measured by gamma-spectroscopy [25], with the previous and the new version of ABRABLA. Obviously, including the production of intermediate-mass fragments by evaporation and simultaneous break-up has essentially improved the agreement with the data in this mass range.

The new accelerator facility FAIR [26], planned by GSI, is dedicated to research with ion and antiproton beams. It will be an outstanding instrument for research on the properties of matter in the dimensions of atoms, nuclei and sub-nuclear particles. The future FAIR facility will provide primary beams with higher intensities and higher energies and a considerably extended variety of secondary beams. In addition, a number of new installations, e.g., powerful magnetic spectrometers, storage rings and colliders will be available, see figure 9. This will also substantially improve the conditions for experiments on nuclear data. A few specific aspects will be presented in more detail.

\section{FAIR}

\subsection{Secondary beams}

Due to the higher beam intensities and a new fragment separator with larger acceptance, the variety of secondary beams will be considerably extended, see figure 10 , providing excellent conditions for research on the properties of exotic nuclei. The secondary beams cover all known nuclides and extend far to the neutron-rich side.

\subsection{The R3B set-up}

The experimental set-up for reaction studies with relativistic radioactive beams (R3B) will be equipped with two magnetic

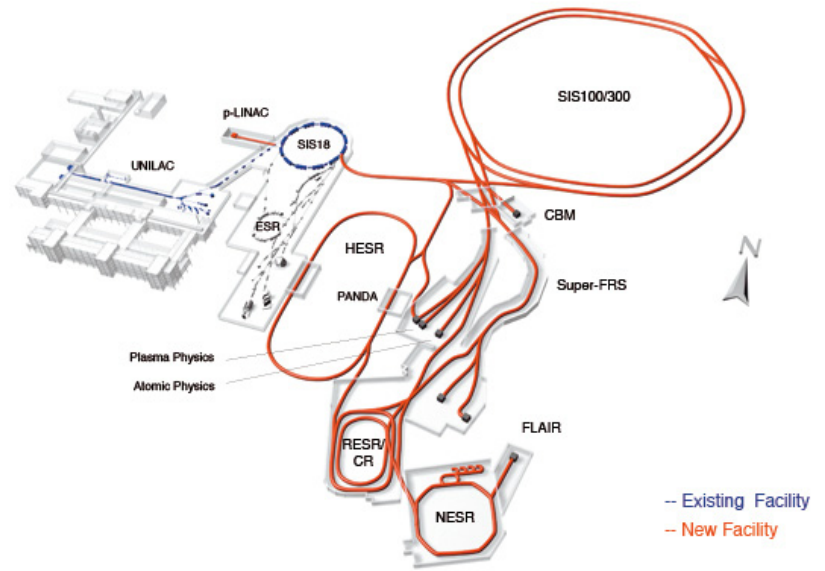

Fig. 9. Installations of the FAIR project of GSI.

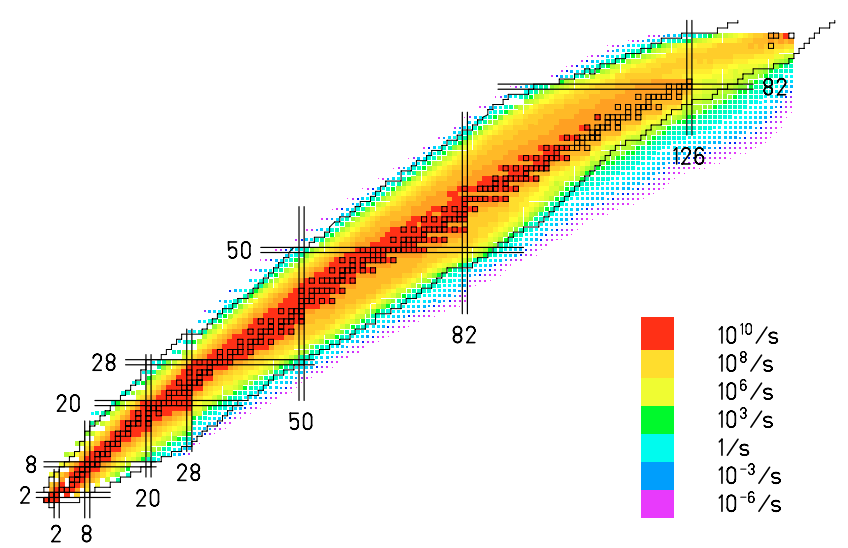

Fig. 10. Estimated intensities of secondary beams at FAIR given in a colour code according to the scale indicated. The full line denotes the limits of nuclides observed up to now.

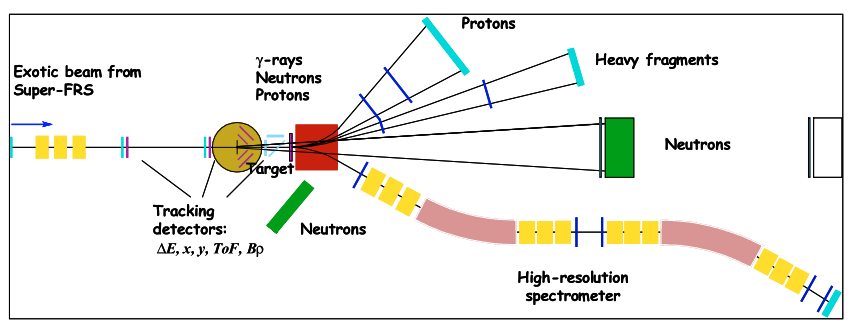

Fig. 11. The R3B experimental set-up.

spectrometers with large acceptance and high resolution, respectively, see figure 11. It will allow for the identification of heavy residues and a simultaneous measurement of neutrons, light charged particles and gammas.

\subsection{Fission experiments in ELISe}

In spite of intensive efforts, the development of fission experiments over many decades has not yet been able to determine the nuclide yields with full resolution in mass number and nuclear charge over the whole mass range for fissioning 


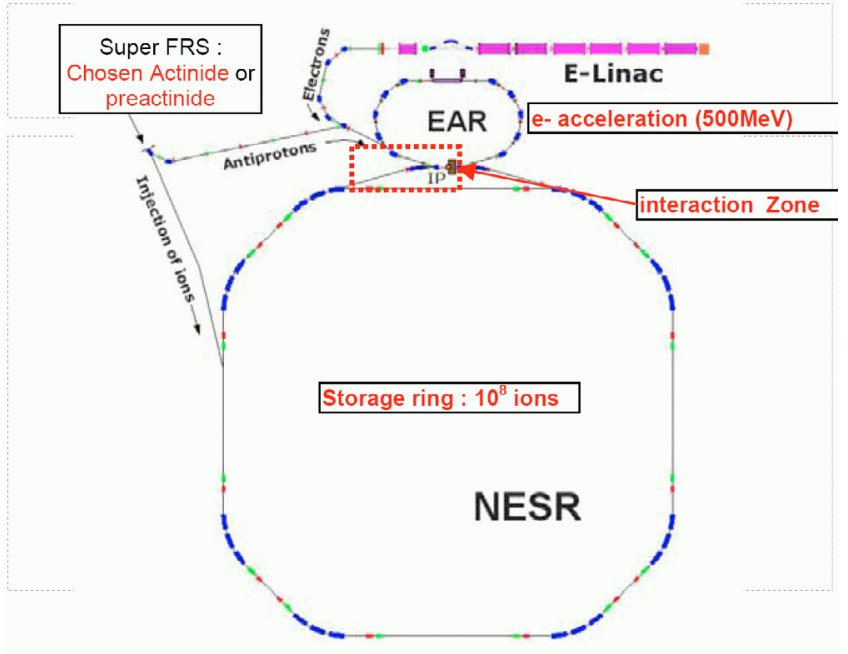

Fig. 12. Schematic view on the electron-ion collider ELISe.

systems with well defined initial conditions. Therefore, there exist absolutely no data on full fission-fragment distributions for specific systems. Fission fragments of the light group could first be fully identified at the Lohengrin spectrograph at ILL, Grenoble with high-resolution energy-loss measurements $[27,28]$. However, these experiments remain limited to thermal-neutron-induced fission. Full nuclide distributions of fission fragments were determined for the first time at the fragment separator of GSI, using relativistic heavy-ion beams [29-31]. This technique, however, was not able to discriminate between the different fissioning systems created in the initial collision with the target. Fission after electromagnetic excitation could be isolated for primary [32] and secondary beams [33], but still the initial excitation energy could not be measured.

The electron-ion collider ELISe [34], depicted in figure 12 , will be the first installation to overcome these problems. Primary or secondary beams will be excited in-flight by inelastic electron collisions. An electron spectrometer and a detection system for the fission fragments will allow measuring the fission-fragment yields of a large number of specific fissioning systems with well defined excitation energy. This will be a major step in the technical development of fission experiments, in particular for establishing the energydependence of microscopic effects in the fission process.

\section{Conclusion}

A comprehensive program on experiments and model developments for a better understanding of spallation reactions is being executed at GSI, Darmstadt. A systematic set of full nuclide distributions has been obtained using the fragment separator as a high-resolution spectrometer. These experiments are complemented by full-acceptance fission experiments and more exclusive experiments emphasizing the detection of lighter fragments.

Progress in modelling the different processes of the spallation reaction has been achieved. The realistic descriptions of spallation-evaporation and spallation-fission have been complemented by sequential binary decay beyond the Businaro-Gallone maximum and simultaneous break-up.

The future FAIR facility will provide substantially improved conditions for the research on a variety of subjects and in particular for experiments on nuclear data. The variety of secondary beams will be considerably extended. The set-up for reaction studies with radioactive beams (R3B) will combine experiments with high resolution and large acceptance. The ELISe electron-ion collider will be the first installation to provide full nuclide distributions for well defined fissioning systems.

The experimental results presented in this contribution have been obtained in a collaboration of CEA Saclay, IPN Orsay, CEN BordeauxGradignan, and GSI Darmstadt. A full list of original publications can be found on the CHARMS Web (http://gsi.de/charms.publica.htm). This work has been supported by the European Union under contracts FIKW-CT-2000-00031, FI6W-516520, FI6W-CT-2004-012985, and FI6W-CT-2004-516352.

\section{References}

1. Yu.A. Litvinov et al., Nucl. Phys. A 756, 3 (2005).

2. T. Kurtukian et al. (these proceedings).

3. K.-H. Schmidt et al., Nucl. Phys. A 665, 221 (2000).

4. A. Kelic et al. (these proceedings).

5. http://www.eurisol.org

6. M.V. Ricciardi et al. (these proceedings).

7. H. Geissel et al., Nucl. Instrum. Meth. B 70, 286 (1992).

8. P. Napolitani et al. (to be published).

9. D. Henzlova et al. (to be published).

10. A.V. Prokofiev, Nucl. Instrum. Meth. A 463, 557 (2001).

11. A.A. Kotov et al., Phys. Rev. C 74, 034605 (2006).

12. K.-H Schmidt et al., Nucl. Phys. A (to be published).

13. B. Fernandez et al., Nucl. Phys. A 747, 227 (2005).

14. M. Bernas et al., Nucl. Phys. A 725, 213 (2003).

15. E. Le Gentil et al., Nucl. Instrum. Meth. A 562, 743 (2006).

16. J.-E. Ducret (these proceedings).

17. A. Boudard et al., Phys. Rev. C 66, 044615 (2002).

18. S. Leray et al., Nucl. Instrum. Meth. A 562, 806 (2006).

19. L.G. Moretto, Nucl. Phys. A 247, 211 (1975).

20. R.J. Charity et al., Nucl. Phys. A 483, 371 (1988).

21. S. Furihata, Nucl. Instrum. Meth. B 171, 251 (2000).

22. R. Bass, Lect. Notes Phys. 117, 281 (1980).

23. M.V. Ricciardi et al., Phys. Rev. C 73, 014607 (2006).

24. K.-H. Schmidt et al. (to be published).

25. Yu.E. Titarenko et al., Nucl. Instrum. Meth. A 562, 801 (2006).

26. http://www.gsi.de/fair/

27. G. Siegert et al., Phys. Lett. B 53, 45 (1970).

28. H.-G. Clerc et al., Nucl. Instrum. Meth. 124, 607 (1975).

29. M. Bernas et al., Phys. Lett. B 331, 19 (1994).

30. P. Armbruster et al., Z. Phys. A 335, 191 (1996).

31. T. Enqvist et al., Nucl. Phys. A 658, 47 (1999).

32. C. Donzaud et al., Eur. Phys. J. A 1, 407 (1998).

33. K.-H. Schmidt et al., Nucl. Phys. A 665, 221 (2000).

34. http://www.gsi.de/fair/experiments/elise/. 